\title{
Association between Dietary Choline and Betaine Intake and 10.6-Year Cardiovascular Disease Outcomes and Mortality in Adults
}

Mahdieh Golzarand ( $\square$ mahdieh_golzarand@yahoo.com )

Tehran University of Medical Sciences https://orcid.org/0000-0003-2651-9276

Parvin Mirmiran

Shahid Beheshti University of Medical Sciences School of Nursing and Midwifery

Fereidoun Azizi

Shahid Beheshti University of Medical Sciences

\section{Research}

Keywords: choline, betaine, cardiovascular disease, stroke, mortality, cohort

Posted Date: July 6th, 2021

DOI: https://doi.org/10.21203/rs.3.rs-639951/v1

License: (c) (i) This work is licensed under a Creative Commons Attribution 4.0 International License.

Read Full License 


\section{Abstract}

Background: Several studies have assessed the association between dietary choline and betaine and cardiovascular disease (CVD) outcomes but their results are inconsistent. The present study aimed to determine the association between dietary intake of choline and betaine and risk of CVD events and mortality in the general population over a 10.6-year of follow-up.

Methods: The present cohort study was conducted on participation in the third wave of the Tehran Lipid and Glucose Study (2006-2008) and was followed-up until March 2018. Dietary intake of choline and betaine was calculated using the United States Department of Agriculture (USDA) database. Data about CVD events and mortality were collected using patients' medical records or death certification.

Results: In this study, 2606 subjects with no previous CVD were participated and followed-up for a median of 10.6 years. During the follow-up periods, 183 incidences of CVD and 17 deaths were detected. Results of the multivariate analysis indicated neither per one standard deviation (SD) increase in dietary choline nor betaine associated with the incidence of CVD, CHD, stroke, and CVD mortality. There was no significant association between food sources of choline and CVD outcomes and mortality. When we assessed the relationship between dietary contributors betaine, per one SD increase in betaine content of fruit and vegetables and oils and fats significantly associated with increased risk of CVD mortality.

Conclusion: Our investigation indicates no association between per one SD increase in choline and betaine and a 10.6-year risk of CVD events and mortality among adults. Besides, we found no relationship between significant food sources of choline and betaine and outcomes of interest.

\section{Introduction}

Cardiovascular disease (CVD) is a global major concern and is the first cause of death in most countries. According to a large study, CVD had affected 422.7 million subjects in the world in 2015 (1) and leads to 17.9 million deaths (one-third of total mortality) every year (2). CVD is comprised of coronary heart disease (CHD), cerebrovascular accident (CVA), peripheral artery disease (PAD), and atrial fibrillation; of those ischemic heart disease and then stroke are the main causes of death worldwide (3) including Iran (4). Important risk factors for CVD are obesity, metabolic disorders, and unhealthy diet, and improvement of these risk factors could reduce the risk of CVD events (5).

Choline is an essential nutrient that is involved in one-carbon metabolism, synthesis of neurotransmitters, and some other functions (6). Choline is synthesized by humans in low values; thus intake of choline from dietary sources including eggs, meat, dairy products, whole grains, and vegetables is important to provide sufficient values of choline and maintenance optimal body functions $(7,8)$. Recently, some studies have reported that a gut microbiota-related metabolite of choline i.e., trimethylamine $\mathrm{N}$-oxide (TMAO), is associated with increased risk of CVD incident and mortality (9-11). Several studies have indicated an inverse association between dietary choline and betaine, a metabolite of choline, with some risk factors of CVD such as inflammatory markers (12) and hyperhomocysteinemia (13); but their results 
on prevention of CVD events are conflicting (14-16). Besides, whether there is a significant association between dietary sources of TMAO i.e., choline and betaine, and risk of CVD and mortality is unclear. Therefore, the present study aimed to determine the association between dietary intake of choline and betaine and risk of CVD events and mortality in the general population over a 10.6-year of follow-up.

\section{Material And Methods}

The present cohort study was conducted using data from subjects who had participated in the cohort of Tehran Lipid and Glucose Study (TLGS). The design and aims of TLGS had been reported previously. In short, 150059 males and females residing district no. 13 of Tehran were enrolled and followed-up once every three years. For the current study, a total of 10091 subjects, aged $\geq 19$ years, who had participated in the third wave of TLGS (2006-2008), were recruited. Eligibility criteria for inclusion were complete data on dietary intakes and demographic characteristics, no history of CVD events at baseline, energy intake between $\geq 2$ Z-scores $\leq$, and complete follow-up. After removing ineligible subjects, our final sample size was included 2606 subjects that were followed-up until March 2018.

This study was conducted according to the Helsinki declaration ethics, written consent was obtained from all participants and the ethical committee of Shahid Beheshti University of Medical Sciences approved the study.

\section{Assessment of covariates}

Routinely, all covariates were measured at baseline and once every three years. Trained interviewers were collected participants' data including age, sex, history of medications, and smoking using a predefined questionnaire. By the same protocol, the level of physical activity was estimated basis on a modifiable activity questionnaire (MAQ) (17) and anthropometric examinations were measured using digital scale (Seca 707, Germany) and tape measure.

Lipid profile and fasting blood glucose (FBG) were measured using fasting blood samples and enzymatic colorimetric tests. Systolic and diastolic blood pressure was determined following 15 minutes rest, in a seated position on the right arm using a mercury sphygmomanometer (Riester, Germany).

\section{Assessment of dietary intake and dietary choline and betaine}

The dietary intake of participants was collected using a validated 168-item food frequency questionnaire (FFQ) (18). For this, the frequency and portion size of each consumed food item during the last 12 months were asked by trained interviewers at baseline. Then foods' portion size was converted to gram and participants' daily intake was calculated.

In the current study, the total amount of dietary choline and betaine from each food item was estimated using the United States Department of Agriculture (USDA) database (19). 


\section{Ascertainment of cardiovascular events and CVD-mortality}

Information about cardiovascular events, mortality, and specific causes of mortality were updated annually by a trained nurse. Then a physician collected patients' medical records or death certification for more evaluation. In the end, the outcome committee of TLGS assessed the outcome data and judged events. CVD events included CHD and stroke.

\section{Statistical analysis}

In the present study dietary choline and betaine were adjusted by the residual model, and their values were categorized as standard deviation (SD). Cox proportional hazards models were applied to explore the relationship between dietary choline and betaine intake and the risk of cardiovascular incidence and CVD mortality. The person-years for each participant were calculated from baseline to the date of first cardiovascular incidence reported, or date of death, or the end of follow-up. Hazard ratio (HR) and 95\% confidence interval $(95 \% \mathrm{Cl})$ were reported for each one SD increase in dietary choline and betaine intake and also food sources of choline and betaine. The first model was adjusted for age and sex. The second model was adjusted for age, sex, total energy intake, smoking, physical activity, obesity (BMI $\geq 30$ $\mathrm{kg} / \mathrm{m} 2$ ), diabetes (FBG $\geq 126 \mathrm{mg} / \mathrm{dl}$ or medications), dyslipidemia ( $\mathrm{TG} \geq 150 \mathrm{mg} / \mathrm{dl}$, or $\mathrm{HDL}<40 \mathrm{mg} / \mathrm{dl}$ in male, $<50 \mathrm{mg} / \mathrm{dl}$ in female, or medications), hypertension (SBP $\geq 140 \mathrm{mmHg}$, or DBP $\geq 90 \mathrm{mmHg}$, or mediations). We also conducted sensitivity analysis by removing high-risk participants to investigate the relationship between per one SD increase in choline and betaine in healthy adults. Data were analyzed using SPSS software (version 20.0; IBM Corporation, Armonk, NY USA). Significant level was defined as P value $<0.05$.

\section{Results}

In the present cohort study, 2606 subjects without CVD history were participants (Fig. 1). The median follow-up period was 10.6 years (IQR: 10.1 to 11.1 years). The median baseline age was 39.0 years and $54.9 \%$ of participants were females. A total of $6.6 \%$ of participants suffered from diabetes, $25.0 \%$ from obesity, $53.1 \%$ from dyslipidemia, and $12.9 \%$ from hypertension. General characteristics of participants are presented in Table 1. 
Table 1

General characteristics of participants

\begin{tabular}{|ll|}
\hline Characteristics & Total cohort $(\mathbf{n}=\mathbf{2 6 0 6})$ \\
\hline Age $($ year $)$ & $39.0(28.0-49.0)$ \\
\hline Male $(\%)$ & $1176(45.1)$ \\
\hline Body mass index $\left(\mathrm{kg} / \mathrm{m}^{2}\right)$ & $26.7(23.7-29.9)$ \\
\hline Fasting blood glucose $(\mathrm{mg} / \mathrm{dl})$ & $87.0(81.0-93.0)$ \\
\hline Total cholesterol $(\mathrm{mg} / \mathrm{dl})$ & $184.0(160.0-212.0)$ \\
\hline Triglyceride $(\mathrm{mg} / \mathrm{dl})$ & $122.0(84.0-178.0)$ \\
\hline High-density cholesterol $(\mathrm{mg} / \mathrm{dl})$ & $41.0(35.0-49.0)$ \\
\hline Low-density cholesterol $(\mathrm{mg} / \mathrm{dl})$ & $113.0(93.2-136.6)$ \\
\hline Systolic blood pressure $(\mathrm{mmHg})$ & $110.0(100.0-120.0)$ \\
\hline Diastolic blood pressure $(\mathrm{mmHg})$ & $73.0(67.0-80.0)$ \\
\hline Smoking $(\%)$ & $242(9.3)$ \\
\hline Physical activity $($ Met-min/wk) & $1000(241-2501)$ \\
\hline $\begin{array}{l}\text { Data presented as median (interquartile range) for continuous and number (\%) for non-continuous } \\
\text { variables }\end{array}$
\end{tabular}

The median dietary intake of choline was $238.5 \mathrm{mg} / \mathrm{d}$ and betaine was $64.9 \mathrm{mg} / \mathrm{d}$. The most important food sources of dietary choline were meat, eggs, and dairy products and for betaine were grains and bakery products (Table 2 ). 
Table 2

Total dietary intake of choline and betaine and their intakes from each food item

\begin{tabular}{|ll|}
\hline Dietary intake & Total cohort $(\mathbf{n = 2 6 0 6 )}$ \\
\hline Dietary energy $(\mathrm{kcal} / \mathrm{d})$ & $2279(1721-2732)$ \\
\hline Energy-adjusted choline $(\mathrm{mg} / \mathrm{d})$ & $238.5(202.0-277.4)$ \\
\hline Energy-adjusted betaine $(\mathrm{mg} / \mathrm{d})$ & $82.1(65.0-102.2)$ \\
\hline Choline-grains and bakery $(\mathrm{mg} / \mathrm{d})$ & $40.7(31.4-51.4)$ \\
\hline Choline-legumes and nuts $(\mathrm{mg} / \mathrm{d})$ & $6.4(3.8-10.1)$ \\
\hline Choline-meat and eggs $(\mathrm{mg} / \mathrm{d})$ & $81.4(58.3-111.9)$ \\
\hline Choline-dairy products $(\mathrm{mg} / \mathrm{d})$ & $43.2(26.3-63.2)$ \\
\hline Choline-fruits and vegetables $(\mathrm{mg} / \mathrm{d})$ & $35.7(25.0-49.7)$ \\
\hline Choline-fats and oils $(\mathrm{mg} / \mathrm{d})$ & $1.8(0.8-2.9)$ \\
\hline Betaine-grains and bakery $(\mathrm{mg} / \mathrm{d})$ & $61.5(43.9-81.8)$ \\
\hline Betaine-legumes and nuts $(\mathrm{mg} / \mathrm{d})$ & $0.3(0.3-0.6)$ \\
\hline Betaine-meat and eggs $(\mathrm{mg} / \mathrm{d})$ & $3.3(2.2-4.7)$ \\
\hline Betaine-dairy products $(\mathrm{mg} / \mathrm{d})$ & $4.8(3.3-6.7)$ \\
\hline Betaine-fruits and vegetables $(\mathrm{mg} / \mathrm{d})$ & $6.7(4.0-10.6)$ \\
\hline Betaine-fats and oils $(\mathrm{mg} / \mathrm{d})$ & $0.005(0.001-0.01)$ \\
\hline Data presented as median $(\mathrm{interquartile}$ range) \\
\hline
\end{tabular}

Over the follow-up periods, there were 183 incidence of CVD (164 CHD and 22 stroke) and 17 deaths incident. Results of multivariate analysis indicated neither per one SD increase in dietary choline nor betaine associated with incidence of CVD (HR: $0.95 ; 95 \% \mathrm{Cl}: 0.79$ to 1.14 and HR: $0.94 ; 95 \% \mathrm{Cl}: 0.81$ to 1.09), CHD (HR: $0.95 ; 95 \% \mathrm{Cl}: 0.81$ to 1.10 and HR: $0.95 ; 95 \% \mathrm{Cl}: 0.81$ to 1.11 ), stroke (HR: $0.82 ; 95 \% \mathrm{Cl}$ : 0.53 to 1.13 and HR: $0.84 ; 95 \% \mathrm{Cl}: 0.56$ to 1.26$)$ and mortality (HR: $1.15 ; 95 \% \mathrm{Cl}: 0.76$ to 1.74 and HR: $1.16 ; 95 \%$ Cl: 0.76 to 1.78 ) (Table 3 ). 
Table 3

Hazard ratios $(95 \% \mathrm{Cl})$ for developing cardiovascular diseases and related mortality per one SD increase in dietary choline and betaine intake

\begin{tabular}{|c|c|c|c|c|}
\hline Dietary intake & CVD & CHD & Stroke & CVD-mortality \\
\hline Person years & 28909 & 28994 & 29760 & 29863 \\
\hline No. of cases & 183 & 164 & 22 & 17 \\
\hline \multicolumn{5}{|c|}{ Choline intake (SD = $94.14 \mathrm{mg} / \mathrm{d})$} \\
\hline $\begin{array}{l}\text { Age and sex-adjusted } \\
\text { model }\end{array}$ & $\begin{array}{l}0.96(0.80- \\
1.16)\end{array}$ & $\begin{array}{l}0.98(0.81- \\
1.19)\end{array}$ & $\begin{array}{l}1.04(0.63- \\
1.72)\end{array}$ & $\begin{array}{l}0.94(0.51- \\
1.75)\end{array}$ \\
\hline $\begin{array}{l}\text { Multivariate-adjusted } \\
\text { model }\end{array}$ & $\begin{array}{l}0.95(0.79- \\
1.14)\end{array}$ & $\begin{array}{l}0.97(0.80- \\
1.18)\end{array}$ & $\begin{array}{l}1.01(0.66- \\
1.56)\end{array}$ & $\begin{array}{l}0.93(0.51- \\
1.69)\end{array}$ \\
\hline \multicolumn{5}{|c|}{ Betaine intake (SD = $36.82 \mathrm{mg} / \mathrm{d})$} \\
\hline $\begin{array}{l}\text { Age and sex-adjusted } \\
\text { model }\end{array}$ & $\begin{array}{l}0.94(0.81- \\
1.09)\end{array}$ & $\begin{array}{l}0.95(0.81- \\
1.10)\end{array}$ & $\begin{array}{l}0.82(0.53- \\
1.13)\end{array}$ & $\begin{array}{l}1.15(0.76- \\
1.74)\end{array}$ \\
\hline $\begin{array}{l}\text { Multivariate-adjusted } \\
\text { model }\end{array}$ & $\begin{array}{l}0.94(0.81- \\
1.09)\end{array}$ & $\begin{array}{l}0.95(0.81- \\
1.11)\end{array}$ & $\begin{array}{l}0.84(0.56- \\
1.26)\end{array}$ & $\begin{array}{l}1.16(0.76- \\
1.78)\end{array}$ \\
\hline \multicolumn{5}{|c|}{$\begin{array}{l}\text { Multivariate-adjusted model adjusted for sex and age, smoking, physical activity, energy intake, } \\
\text { obesity, diabetes, dyslipidemia, hypertension. }\end{array}$} \\
\hline \multicolumn{5}{|c|}{ BMI: body mass index; CHD: coronary heart disease; CVD: cardiovascular disease } \\
\hline
\end{tabular}

Results of sensitivity analysis with choline, neither show statistically significant association with cardiovascular events nor death were detected among healthy adults. In addition, individual analyses for betaine were not significant (Table 4). 
Table 4

Hazard ratios $(95 \% \mathrm{Cl})$ for developing cardiovascular diseases and related mortality per one SD increase in dietary choline and betaine intake among healthy subjects and high-risk patients

$\begin{array}{lllll}\text { Dietary intake } & \text { CVD } & \text { CHD } & \text { Stroke } & \text { CVD-mortality }\end{array}$

Healthy subjects $(n=909)$

Choline intake $(S D=89.12 \mathrm{mg} / \mathrm{d})$

\begin{tabular}{llll}
$\begin{array}{lll}\text { Age and sex-adjusted } \\
\text { model }\end{array}$ & $0.93(0.51-$ & $0.90(0.64-$ & $1.11(0.30-$ \\
& $1.68)$ & $1.75)$ & $4.03)$ \\
\hline $\begin{array}{l}\text { Multivariate-adjusted } \\
\text { model }^{\dagger}\end{array}$ & $\begin{array}{l}0.88(0.47- \\
1.64)\end{array}$ & $\begin{array}{l}0.85(0.40- \\
1.77)\end{array}$ & $1.01(0.33-$ \\
\hline
\end{tabular}

\section{Betaine intake $(S D=44.89 \mathrm{mg} / \mathrm{d})$}

\begin{tabular}{llll}
$\begin{array}{lll}\text { Age and sex-adjusted } \\
\text { model }\end{array}$ & $0.98(0.57-$ & $0.81(0.42-$ & $1.69(0.73-$ \\
& $1.67)$ & $1.53)$ & $3.91)$ \\
\hline $\begin{array}{l}\text { Multivariate-adjusted } \\
\text { model }^{\dagger}\end{array}$ & $\begin{array}{l}0.92(0.51- \\
1.68)\end{array}$ & $\begin{array}{l}0.79(0.39- \\
1.61)\end{array}$ & $1.27(0.47-$ \\
\hline
\end{tabular}

High-risk patients $(n=1697)$

Choline intake $(S D=96.69 \mathrm{mg} / \mathrm{d})$

\begin{tabular}{lllll}
$\begin{array}{l}\text { Age and sex-adjusted } \\
\text { model }\end{array}$ & $0.98(0.80-$ & $1.00(0.82-$ & $1.07(0.66-$ & $0.96(0.51-$ \\
& $1.18)$ & $1.22)$ & $1.73)$ & $1.78)$ \\
\hline $\begin{array}{l}\text { Multivariate-adjusted } \\
\text { model }^{\ddagger}\end{array}$ & $\begin{array}{l}0.99(0.82- \\
1.20)\end{array}$ & $1.02(0.84-$ & $1.03(0.66-$ & $0.99(0.55-$ \\
\hline
\end{tabular}

Betaine intake $(S D=49.26 \mathrm{mg} / \mathrm{d})$

\begin{tabular}{|c|c|c|c|c|}
\hline $\begin{array}{l}\text { Age and sex-adjusted } \\
\text { model }\end{array}$ & $\begin{array}{l}0.96(0.82- \\
1.13)\end{array}$ & $\begin{array}{l}0.96(0.81- \\
1.14)\end{array}$ & $\begin{array}{l}0.99(0.63- \\
1.58)\end{array}$ & $\begin{array}{l}1.19(0.80- \\
1.78)\end{array}$ \\
\hline $\begin{array}{l}\text { Multivariate-adjusted } \\
\text { model }^{\ddagger}\end{array}$ & $\begin{array}{l}0.91(0.75- \\
1.12)\end{array}$ & $\begin{array}{l}0.93(0.79- \\
1.15)\end{array}$ & $\begin{array}{l}0.74(0.40- \\
1.36)\end{array}$ & $\begin{array}{l}1.20(0.69- \\
2.00)\end{array}$ \\
\hline
\end{tabular}

${ }^{+}$Multivariate-adjusted model adjusted for sex and age, smoking, physical activity, energy intake.

F Multivariate-adjusted model adjusted for sex and age, smoking, physical activity, energy intake, BMI, FBG, lipid profile, blood pressure.

BMI: body mass index; CHD: coronary heart disease; CVD: cardiovascular disease

Association between dietary contributors of choline and betaine with the outcome of interest is presented in Tables 5 and 6 . There was no significant association between food sources of choline and CVD outcomes and mortality. When we assessed the relationship between dietary contributors betaine, per one SD increase in betaine content of fruit and vegetables (HR: $1.44 ; 95 \% \mathrm{Cl}: 1.07$ to 1.93 ) and oils and fats (HR: $1.31 ; 95 \%$ Cl: 1.11 to 1.54 ) significantly associated with increased risk of CVD-mortality. 
Table 5

Hazard ratios $(95 \% \mathrm{Cl})$ for developing cardiovascular diseases and related mortality per one SD increase in choline food sources

\begin{tabular}{|c|c|c|c|c|}
\hline & CVD & CHD & Stroke & CVD-mortality \\
\hline \multicolumn{5}{|c|}{ Dairy products (SD = $31.30 \mathrm{mg} / \mathrm{d}$ ) } \\
\hline $\begin{array}{l}\text { Age and sex-adjusted } \\
\text { model }\end{array}$ & $\begin{array}{l}0.94(0.80- \\
1.10)\end{array}$ & $\begin{array}{l}0.95(0.80- \\
1.13)\end{array}$ & $\begin{array}{l}0.67(0.39- \\
1.15)\end{array}$ & $\begin{array}{l}0.93(0.54- \\
1.57)\end{array}$ \\
\hline $\begin{array}{l}\text { Multivariate-adjusted } \\
\text { model }\end{array}$ & $\begin{array}{l}0.93(0.79- \\
1.08)\end{array}$ & $\begin{array}{l}0.94(0.80- \\
1.11)\end{array}$ & $\begin{array}{l}0.69(0.41- \\
1.13)\end{array}$ & $\begin{array}{l}0.89(0.54- \\
1.48)\end{array}$ \\
\hline \multicolumn{5}{|c|}{ Meats and eggs (SD = $57.77 \mathrm{mg} / \mathrm{d})$} \\
\hline $\begin{array}{l}\text { Age and sex-adjusted } \\
\text { model }\end{array}$ & $\begin{array}{l}0.98(0.83- \\
1.15)\end{array}$ & $\begin{array}{l}0.97(0.82- \\
1.15)\end{array}$ & $\begin{array}{l}1.01(0.63- \\
1.60)\end{array}$ & $\begin{array}{l}1.07(0.67- \\
1.73)\end{array}$ \\
\hline $\begin{array}{l}\text { Multivariate-adjusted } \\
\text { model }\end{array}$ & $\begin{array}{l}0.98(0.84- \\
1.15)\end{array}$ & $\begin{array}{l}0.97(0.82- \\
1.15)\end{array}$ & $\begin{array}{l}1.01(0.68- \\
1.52)\end{array}$ & $\begin{array}{l}1.06(0.68- \\
1.64)\end{array}$ \\
\hline \multicolumn{5}{|c|}{ Grains and bakery products (SD = $20.58 \mathrm{mg} / \mathrm{d}$ ) } \\
\hline $\begin{array}{l}\text { Age and sex-adjusted } \\
\text { model }\end{array}$ & $\begin{array}{l}0.94(0.82- \\
1.07)\end{array}$ & $\begin{array}{l}0.95(0.82- \\
1.09)\end{array}$ & $\begin{array}{l}0.80(0.52- \\
1.23)\end{array}$ & $\begin{array}{l}0.97(0.63- \\
1.49)\end{array}$ \\
\hline $\begin{array}{l}\text { Multivariate-adjusted } \\
\text { model }\end{array}$ & $\begin{array}{l}0.93(0.81- \\
1.07)\end{array}$ & $\begin{array}{l}0.94(0.81- \\
1.09)\end{array}$ & $\begin{array}{l}0.82(0.55- \\
1.22)\end{array}$ & $\begin{array}{l}0.97(0.63- \\
1.48)\end{array}$ \\
\hline \multicolumn{5}{|c|}{ Fruits and vegetables $(S D=22.13 \mathrm{mg} / \mathrm{d}$ ) } \\
\hline $\begin{array}{l}\text { Age and sex-adjusted } \\
\text { model }\end{array}$ & $\begin{array}{l}1.05(0.90- \\
1.22)\end{array}$ & $\begin{array}{l}1.03(0.88- \\
1.22)\end{array}$ & $\begin{array}{l}1.22(0.83- \\
1.80)\end{array}$ & $\begin{array}{l}1.15(0.71- \\
1.84)\end{array}$ \\
\hline $\begin{array}{l}\text { Multivariate-adjusted } \\
\text { model }\end{array}$ & $\begin{array}{l}1.03(0.88- \\
1.20)\end{array}$ & $\begin{array}{l}1.02(0.86- \\
1.20)\end{array}$ & $\begin{array}{l}1.25(0.76- \\
1.65)\end{array}$ & $\begin{array}{l}1.14(0.73- \\
1.80)\end{array}$ \\
\hline \multicolumn{5}{|c|}{ Nuts and legumes (SD = $9.77 \mathrm{mg} / \mathrm{d})$} \\
\hline $\begin{array}{l}\text { Age and sex-adjusted } \\
\text { model }\end{array}$ & $\begin{array}{l}1.03(0.88- \\
1.21)\end{array}$ & $\begin{array}{l}1.06(0.90- \\
1.24)\end{array}$ & $\begin{array}{l}1.14(0.77- \\
1.68)\end{array}$ & $\begin{array}{l}0.88(0.45- \\
1.71)\end{array}$ \\
\hline $\begin{array}{l}\text { Multivariate-adjusted } \\
\text { model }\end{array}$ & $\begin{array}{l}1.03(0.86- \\
1.22)\end{array}$ & $\begin{array}{l}1.05(0.88- \\
1.26)\end{array}$ & $\begin{array}{l}1.12(0.78- \\
1.61)\end{array}$ & $\begin{array}{l}0.85(0.43- \\
1.67)\end{array}$ \\
\hline \multicolumn{5}{|c|}{ Fats and oils (SD = $1.88 \mathrm{mg} / \mathrm{d})$} \\
\hline $\begin{array}{l}\text { Age and sex-adjusted } \\
\text { model }\end{array}$ & $\begin{array}{l}1.03(0.89- \\
1.19)\end{array}$ & $\begin{array}{l}1.07(0.92- \\
1.24)\end{array}$ & $\begin{array}{l}0.78(0.47- \\
1.32)\end{array}$ & $\begin{array}{l}1.26(0.87- \\
1.81)\end{array}$ \\
\hline $\begin{array}{l}\text { Multivariate-adjusted } \\
\text { model }\end{array}$ & $\begin{array}{l}1.06(0.92- \\
1.23)\end{array}$ & $\begin{array}{l}1.10(0.95- \\
1.29)\end{array}$ & $\begin{array}{l}0.85(0.53- \\
1.37)\end{array}$ & $\begin{array}{l}1.34(0.91- \\
1.97)\end{array}$ \\
\hline
\end{tabular}




\begin{tabular}{|c|c|c|}
\hline CVD & Stroke & CVD-mortality \\
\hline \multicolumn{3}{|c|}{$\begin{array}{l}\text { Multivariate-adjusted model adjusted for sex and age, smoking, physical activity, energy intake, } \\
\text { obesity (BMI } \geq 30 \mathrm{~kg} / \mathrm{m} 2) \text {, diabetes (FBG } \geq 126 \mathrm{mg} / \mathrm{dl} \text { or take medications), dyslipidemia (TG } \geq 150 \\
\mathrm{mg} / \mathrm{dl}, \text { or } \mathrm{HDL}<40 \mathrm{mg} / \mathrm{dl}(\mathrm{male}), 50 \mathrm{mg} / \mathrm{dl} \text { (female), or medications), hypertension (SBP } \geq 14 \mathrm{mmHg} \text {, } \\
\text { or } D B P \geq 9 \mathrm{mmHg} \text {, or mediations) }\end{array}$} \\
\hline \multicolumn{3}{|c|}{$\begin{array}{l}\text { BMI: body mass index; CHD: coronary heart disease; CVD: cardiovascular disease; DBP: diastolic } \\
\text { blood pressure; FBG: fasting blood glucose; HDL: high-density cholesterol; SBP: systolic blood } \\
\text { pressure; SD: standard deviation; TG: triglyceride. }\end{array}$} \\
\hline
\end{tabular}


Table 6

Hazard ratios $(95 \% \mathrm{Cl})$ for developing cardiovascular diseases and related mortality per one SD increase in betaine food sources

\begin{tabular}{|c|c|c|c|c|}
\hline & CVD & CHD & Stroke & CVD-mortality \\
\hline \multicolumn{5}{|c|}{ Dairy products (SD = $4.39 \mathrm{mg} / \mathrm{d}$ ) } \\
\hline $\begin{array}{l}\text { Age and sex-adjusted } \\
\text { model }\end{array}$ & $\begin{array}{l}1.03(0.88- \\
1.21)\end{array}$ & $\begin{array}{l}1.03(0.88- \\
1.22)\end{array}$ & $\begin{array}{l}0.95(0.55- \\
1.63)\end{array}$ & $\begin{array}{l}0.87(0.45- \\
1.68)\end{array}$ \\
\hline $\begin{array}{l}\text { Multivariate-adjusted } \\
\text { model }\end{array}$ & $\begin{array}{l}1.07(0.93- \\
1.22)\end{array}$ & $\begin{array}{l}1.07(0.93- \\
1.24)\end{array}$ & $\begin{array}{l}1.02(0.66- \\
1.57)\end{array}$ & $\begin{array}{l}0.92(0.50- \\
1.71)\end{array}$ \\
\hline \multicolumn{5}{|c|}{ Meats and eggs $(\mathrm{SD}=2.96 \mathrm{mg} / \mathrm{d})$} \\
\hline $\begin{array}{l}\text { Age and sex-adjusted } \\
\text { model }\end{array}$ & $\begin{array}{l}0.96(0.82- \\
1.12)\end{array}$ & $\begin{array}{l}0.97(0.82- \\
1.14)\end{array}$ & $\begin{array}{l}0.83(0.49- \\
1.41)\end{array}$ & $\begin{array}{l}0.95(0.56- \\
1.60)\end{array}$ \\
\hline $\begin{array}{l}\text { Multivariate-adjusted } \\
\text { model }\end{array}$ & $\begin{array}{l}0.94(0.80- \\
1.10)\end{array}$ & $\begin{array}{l}0.95(0.80- \\
1.13)\end{array}$ & $\begin{array}{l}0.83(0.52- \\
1.32)\end{array}$ & $\begin{array}{l}0.98(0.59- \\
1.62)\end{array}$ \\
\hline \multicolumn{5}{|c|}{ Grains and bakery products (SD = $37.64 \mathrm{mg} / \mathrm{d})$} \\
\hline $\begin{array}{l}\text { Age and sex-adjusted } \\
\text { model }\end{array}$ & $\begin{array}{l}0.93(0.80- \\
1.08)\end{array}$ & $\begin{array}{l}0.93(0.80- \\
1.09)\end{array}$ & $\begin{array}{l}0.85(0.54- \\
1.32)\end{array}$ & $\begin{array}{l}1.06(0.68- \\
1.64)\end{array}$ \\
\hline $\begin{array}{l}\text { Multivariate-adjusted } \\
\text { model }\end{array}$ & $\begin{array}{l}0.93(0.80- \\
1.08)\end{array}$ & $\begin{array}{l}0.93(0.79- \\
1.09)\end{array}$ & $\begin{array}{l}0.84(0.56- \\
1.26)\end{array}$ & $\begin{array}{l}1.13(0.71- \\
1.81)\end{array}$ \\
\hline \multicolumn{5}{|c|}{ Fruits and vegetables $(S D=8.73 \mathrm{mg} / \mathrm{d}$ ) } \\
\hline $\begin{array}{l}\text { Age and sex-adjusted } \\
\text { model }\end{array}$ & $\begin{array}{l}1.05(0.91- \\
1.21)\end{array}$ & $\begin{array}{l}1.07(0.92- \\
1.23)\end{array}$ & $\begin{array}{l}0.83(0.49- \\
1.41)\end{array}$ & $\begin{array}{l}1.39(1.06- \\
1.82)\end{array}$ \\
\hline $\begin{array}{l}\text { Multivariate-adjusted } \\
\text { model }\end{array}$ & $\begin{array}{l}1.07(0.93- \\
1.24)\end{array}$ & $\begin{array}{l}1.10(0.95- \\
1.28)\end{array}$ & $\begin{array}{l}0.84(0.52- \\
1.37)\end{array}$ & $\begin{array}{l}1.44(1.07- \\
1.93)\end{array}$ \\
\hline \multicolumn{5}{|c|}{ Nuts and legumes (SD = $1.35 \mathrm{mg} / \mathrm{d})$} \\
\hline $\begin{array}{l}\text { Age and sex-adjusted } \\
\text { model }\end{array}$ & $\begin{array}{l}1.03(0.92- \\
1.14)\end{array}$ & $\begin{array}{l}1.04(0.93- \\
1.16)\end{array}$ & $\begin{array}{l}1.19(1.00- \\
1.41)\end{array}$ & $\begin{array}{l}0.32(0.07- \\
1.38)\end{array}$ \\
\hline $\begin{array}{l}\text { Multivariate-adjusted } \\
\text { model }\end{array}$ & $\begin{array}{l}1.04(0.93- \\
1.16)\end{array}$ & $\begin{array}{l}1.05(0.94- \\
1.17)\end{array}$ & $\begin{array}{l}1.15(0.97- \\
1.37)\end{array}$ & $\begin{array}{l}0.23(0.02- \\
2.11)\end{array}$ \\
\hline \multicolumn{5}{|c|}{ Fats and oils (SD = $0.03 \mathrm{mg} / \mathrm{d})$} \\
\hline $\begin{array}{l}\text { Age and sex-adjusted } \\
\text { model }\end{array}$ & $\begin{array}{l}1.03(0.89- \\
1.20)\end{array}$ & $\begin{array}{l}1.06(0.92- \\
1.22)\end{array}$ & $\begin{array}{l}0.58(0.17- \\
1.94)\end{array}$ & $\begin{array}{l}1.28(1.10- \\
1.49)\end{array}$ \\
\hline $\begin{array}{l}\text { Multivariate-adjusted } \\
\text { model }\end{array}$ & $\begin{array}{l}1.03(0.89- \\
1.20)\end{array}$ & $\begin{array}{l}1.06(0.92- \\
1.23)\end{array}$ & $\begin{array}{l}0.68(0.27- \\
1.70)\end{array}$ & $\begin{array}{l}1.31(1.11- \\
1.54)\end{array}$ \\
\hline
\end{tabular}




\section{CVD}

CHD

Stroke

CVD-mortality

Multivariate-adjusted model adjusted for sex and age, smoking, physical activity, energy intake, obesity (BMI $\geq 30 \mathrm{~kg} / \mathrm{m} 2$ ), diabetes ( $F B G \geq 126 \mathrm{mg} /$ dl or take medications), dyslipidemia ( $T G \geq 150$ $\mathrm{mg} / \mathrm{dl}$, or $\mathrm{HDL}<40 \mathrm{mg} / \mathrm{dl}$ (male), $50 \mathrm{mg} / \mathrm{dl}$ (female), or medications), hypertension (SBP $\geq 14 \mathrm{mmHg}$, or $D B P \geq 9 \mathrm{mmHg}$, or mediations)

BMI: body mass index; CHD: coronary heart disease; CVD: cardiovascular disease; DBP: diastolic blood pressure; FBG: fasting blood glucose; HDL: high-density cholesterol; SBP: systolic blood pressure; SD: standard deviation; TG: triglyceride.

\section{Discussion}

In the present study, dietary choline and betaine intake did not associate with the risk of CVD events and mortality in the general population. After sensitivity analysis, no significant association between per one SD increased in choline and betaine and cardiovascular events and death was found among healthy adults and high-risk patients of CVD. Besides, there was no relationship between food sources of choline and betaine and outcomes of interest.

This study was not the first to report that there is no significant link between dietary choline and betaine and CVD outcomes in the general population. Data of 16165 women free from CVD who participated in the PROSPECT-EPIC cohort, revealed no significant relationship between dietary choline and betaine and risk of total CVD, CHD, and CAD over eight years of follow-up (20). Results of the Atherosclerosis Risk in Communities (ARIC) study, in 14430 adults with no CHD at baseline, indicated that 14-year CHD incident in the highest quartile of choline (HR: $1.22,95 \%$ Cl: 0.91 to 1.64) and choline with betaine (HR: 1.14, $95 \%$ Cl: 0.85 to 1.53 ) is comparable with the lowest quartile of intake (16). In the Jackson Heart Study, choline consumption is inversely associated with stroke incidents $(\beta=-0.33)$. But after post hoc analysis, the incidence of stroke across quartiles of dietary choline was not significant (14). In another related study conducted by Nagata et al. (21), there was no significant relationship between choline and CVD mortality among Japanese males and females.

On contrary, Mazidi et al. (22) in a prospective study (6.5-year of follow-up) reported that subject in the quartile of choline had $23 \%$ (95\% Cl: 1.09 to 1.38) higher risk of all cause-mortality, 33\% (95\% Cl: 1.19 to 1.48) CVD mortality and $30 \%(95 \% \mathrm{Cl}: 1.02$ to 1.66$)$ stroke mortality than those in the first quartile. Millar et al. (14) also showed betaine intake increased the risk of CHD by $89 \%$ in the third quartile of intake compared with the lowest quartile. While in the Takayama study, dietary intake of betaine was inversely linked to the CHD mortality among Japanese males (21).

Although all these studies had prospective designs; but their conflicting results may be related to different sample sizes, various follow-up periods, and disparity in subjects' geographical location and race or residual confounders. Besides, inconsistency in findings may be attributed to diversity in food sources of choline and betaine among various populations and distinct bioavailability of choline and betaine from separate foods. In the present study, the same as Bidulescu et al. (16) who reported no link between 
choline and betaine and CVD events, meat and eggs were major food sources of choline, and grains and bakery products were for betaine. In Nagata et al. (21) also eggs were the most contributor of choline. But the seafood was the main food supplying betaine that may explain an inverse association between betaine and mortality from CHD. Seafood is the major source of omega-3 polyunsaturated fatty acid (PUFA), which has been shown to protect against CVD in many studies (23-25). A recent meta-analysis reported an inverse association between fish consumption and risk of $\mathrm{CHD}$ mortality (RR: $0.85,95 \% \mathrm{Cl}$ : 0.77 to 0.94 ), results of this study revealed each $20 \mathrm{~g}$ of fish consumed per day reducing CHD mortality by $4 \%$ (RR: $0.96,95 \% \mathrm{Cl}: 0.95$ to 0.97$)(26)$.

In addition, we evaluated the association between each food source of choline with the risk of CVD events and mortality, with null findings. As motioned above, in our study, meat and eggs were major food sources of choline. In the past studies, the link between food sources of choline and outcomes of interest did not investigate. Mei et al. (27) in a cross-sectional study on 3973 adults indicated fish, red meat and eggs were the most dietary factors that significantly increased TMAO production. However, they noted that the association between red meat and TMAO is due to TMA production from carnitine. Their findings were in agreement with the results of a clinical trial that showed carnitine content of red meat increased production of TMAO but not choline (28). These surveys supported a null association between meat and eggs with CVD in the current study.

Among dietary contributors betaine, we observed a positive link between fruits and vegetables and oils and fats intake and death from CVD. It is unclear why the betaine content of fruits and vegetables is responsible for the increased CVD mortality, but in our study fruits and vegetables and oils and fats are insignificant food sources of betaine.

Furthermore, differences in results of studies may be due to variations in gut microbiota composition across populations. TMAO is formed by the gut microbiota and is positively associated with CVD. Results of a new study indicated a significant relationship between nine gut bacterial species and TMAO (27). Therefore, differences in the gut microbiota of subjects may have an impact on TMAO development. Several factors such as dietary habits, lifestyle, and health status have an impact on gut microbiota composition. In the present study, we stratified subjects into two groups: healthy subjects and patients at high-risk of CVD then re-analyzed data, but the results of the study remained unchanged. Previous studies did not evaluate the relationship between dietary choline and betaine and CVD outcomes among high-risk patients. Hence further investigations to assess whether gut microbiota plays a role in the association between dietary choline and CVD events are warranted.

There are several strengths and limitations. The follow-up period was extended. Outcomes were not selfreported and collected based on medical records. FFQ applied in our study was also validated to assess dietary intake of foods that are the source of choline and betaine. In addition, we assessed the association between each food source of choline and betaine with outcomes of interest. However, the main limitation was that dietary intake of choline and betaine were collected at baseline and they might 
be changed over the follow-up. Furthermore, residual confounders cannot be ruled out even after adjusting for possible confounders in the studies.

\section{Conclusion}

In conclusion, our investigation indicates no significant association between per one SD increase in dietary intake of choline and betaine and a 10.6-year risk of CVD events and mortality among adults. Results of sensitivity analysis reveal the observed relationship did not differ between healthy adults and high-risk patients of CVD. Besides, we found no relationship between significant food sources of choline and betaine and outcomes of interest.

\section{Abbreviations}

CHD: Coronary heart disease

CVA: Cerebrovascular accident

CVD: Cardiovascular disease

FBG: Fasting blood glucose

FFQ: Food frequency questionnaire

MAQ: Modifiable activity questionnaire

PAD: Peripheral artery disease

SD: Standard deviation

TLGS: Tehran Lipid and Glucose Study

TMAO: Trimethylamine $\mathrm{N}$-oxide

USDA: United States Department of Agriculture

\section{Declarations}

Ethics approval and consent to participate: This study was conducted according to the Helsinki declaration ethics, written consent was obtained from all participants and the ethical committee of Shahid Beheshti University of Medical Sciences approved the study.

Consent for publication: Not applicable.

Availability of data and materials: Not applicable. 
Funding: Not applicable.

Competing interests: The authors declare that there is no conflict of interest.

Authors' contribution: M.G and P.M participated in conception and design of study. M.G participated analyzed and interpreted data. M.G, P.M prepared draft of manuscript. All authors read and approved the manuscript.

Acknowledgements: Not applicable.

\section{References}

1. Roth GA, Johnson C, Abajobir A, Abd-Allah F, Abera SF, Abyu G, et al. Global, Regional, and National Burden of Cardiovascular Diseases for 10 Causes, 1990 to 2015. J Am Coll Cardiol. 2017,70(1):1-25.

2. World Health Organization. Cardiovascular Diseases. 2020. (https://www.who.int/healthtopics/cardiovascular-diseases/\#tab=tab_1).

3. Hinton W, McGovern A, Coyle R, Han TS, Sharma P, Correa A, et al. Incidence and prevalence of cardiovascular disease in English primary care: a cross-sectional and follow-up study of the Royal College of General Practitioners (RCGP) Research and Surveillance Centre (RSC). BMJ open. 2018,8(8):e020282.

4. Sarrafzadegan N, Mohammmadifard N. Cardiovascular Disease in Iran in the Last 40 Years: Prevalence, Mortality, Morbidity, Challenges and Strategies for Cardiovascular Prevention. Archives of Iranian medicine. 2019,22(4):204-10.

5. Yusuf S, Joseph P, Rangarajan S, Islam S, Mente A, Hystad P, et al. Modifiable risk factors, cardiovascular disease, and mortality in 155722 individuals from 21 high-income, middle-income, and low-income countries (PURE): a prospective cohort study. Lancet (London, England). 2020,395(10226):795-808.

6. Leermakers ET, Moreira EM, Kiefte-de Jong JC, Darweesh SK, Visser T, Voortman T, et al. Effects of choline on health across the life course: a systematic review. Nutrition reviews. 2015,73(8):500-22.

7. Rajaie S, Esmaillzadeh A. Dietary choline and betaine intakes and risk of cardiovascular diseases: review of epidemiological evidence. ARYA atherosclerosis. 2011,7(2):78-86.

8. Meyer KA, Shea JW. Dietary Choline and Betaine and Risk of CVD: A Systematic Review and MetaAnalysis of Prospective Studies. Nutrients. 2017,9(7).

9. Naghipour S, Cox AJ, Peart JN, Du Toit EF, Headrick JP. Trimethylamine-N-Oxide: Heart of the microbiota-cardiovascular disease nexus? Nutrition research reviews. 2020:1-64.

10. Heianza Y, Ma W, Manson JE, Rexrode KM, Qi L. Gut Microbiota Metabolites and Risk of Major Adverse Cardiovascular Disease Events and Death: A Systematic Review and Meta-Analysis of Prospective Studies. Journal of the American Heart Association. 2017,6(7). 
11. Schiattarella GG, Sannino A, Toscano E, Giugliano G, Gargiulo G, Franzone A, et al. Gut microbegenerated metabolite trimethylamine-N-oxide as cardiovascular risk biomarker: a systematic review and dose-response meta-analysis. European heart journal. 2017,38(39):2948-56.

12. Detopoulou P, Panagiotakos DB, Antonopoulou S, Pitsavos C, Stefanadis C. Dietary choline and betaine intakes in relation to concentrations of inflammatory markers in healthy adults: the ATTICA study. The American journal of clinical nutrition. 2008,87(2):424-30.

13. Ueland PM. Choline and betaine in health and disease. Journal of inherited metabolic disease. 2011,34(1):3-15.

14. Millard HR, Musani SK, Dibaba DT, Talegawkar SA, Taylor HA, Tucker KL, et al. Dietary choline and betaine, associations with subclinical markers of cardiovascular disease risk and incidence of CVD, coronary heart disease and stroke: the Jackson Heart Study. European journal of nutrition. 2018,57(1):51-60.

15. Bertoia ML, Pai JK, Cooke JP, Joosten MM, Mittleman MA, Rimm EB, et al. Plasma homocysteine, dietary $\mathrm{B}$ vitamins, betaine, and choline and risk of peripheral artery disease. Atherosclerosis. 2014,235(1):94-101.

16. Bidulescu A, Chambless LE, Siega-Riz AM, Zeisel SH, Heiss G. Usual choline and betaine dietary intake and incident coronary heart disease: the Atherosclerosis Risk in Communities (ARIC) study. BMC cardiovascular disorders. 2007,7:20.

17. Kriska AM, Knowler WC, LaPorte RE, Drash AL, Wing RR, Blair SN, et al. Development of questionnaire to examine relationship of physical activity and diabetes in Pima Indians. Diabetes care. 1990,13(4):401-11.

18. Esfahani FH, Asghari G, Mirmiran P, Azizi F. Reproducibility and relative validity of food group intake in a food frequency questionnaire developed for the Tehran Lipid and Glucose Study. Journal of epidemiology. 2010,20(2):150-8.

19. Patterson KY, Bhagwat SA, Williams JR, Howe JC, Holden J, Zeisel S, et al. USDA database for the choline content of common foods, release two. Nutrient Data Laboratory, Beltsville Human Nutrition Research Center, ARS, USDA. 2008.

20. Dalmeijer GW, Olthof MR, Verhoef P, Bots ML, van der Schouw YT. Prospective study on dietary intakes of folate, betaine, and choline and cardiovascular disease risk in women. European journal of clinical nutrition. 2008,62(3):386-94.

21. Nagata C, Wada K, Tamura T, Konishi K, Kawachi T, Tsuji M, et al. Choline and Betaine Intakes Are Not Associated with Cardiovascular Disease Mortality Risk in Japanese Men and Women. The Journal of nutrition. 2015,145(8):1787-92.

22. Mazidi M, Katsiki N, Mikhailidis DP, Banach M. Dietary choline is positively related to overall and cause-specific mortality: results from individuals of the National Health and Nutrition Examination Survey and pooling prospective data. The British journal of nutrition. 2019,122(11):1262-70.

23. de Goede J, Geleijnse JM, Boer JM, Kromhout D, Verschuren WM. Marine (n-3) fatty acids, fish consumption, and the 10-year risk of fatal and nonfatal coronary heart disease in a large population 
of Dutch adults with low fish intake. The Journal of nutrition. 2010,140(5):1023-8.

24. Mozaffarian D, Lemaitre RN, Kuller LH, Burke GL, Tracy RP, Siscovick DS. Cardiac benefits of fish consumption may depend on the type of fish meal consumed: the Cardiovascular Health Study. Circulation. 2003,107(10):1372-7.

25. Hu FB, Bronner L, Willett WC, Stampfer MJ, Rexrode KM, Albert CM, et al. Fish and omega-3 fatty acid intake and risk of coronary heart disease in women. Jama. 2002,287(14):1815-21.

26. Zhang B, Xiong K, Cai J, Ma A. Fish Consumption and Coronary Heart Disease: A Meta-Analysis. Nutrients. 2020,12(8).

27. Mei Z, Chen GC, Wang Z, Usyk M, Yu B, Baeza YV, et al. Dietary factors, gut microbiota, and serum trimethylamine-N-oxide associated with cardiovascular disease in the Hispanic Community Health Study/Study of Latinos. The American journal of clinical nutrition. 2021.

28. Wang Z, Bergeron N, Levison BS, Li XS, Chiu S, Jia X, et al. Impact of chronic dietary red meat, white meat, or non-meat protein on trimethylamine $\mathrm{N}$-oxide metabolism and renal excretion in healthy men and women. European heart journal. 2019,40(7):583-94.

\section{Figures}

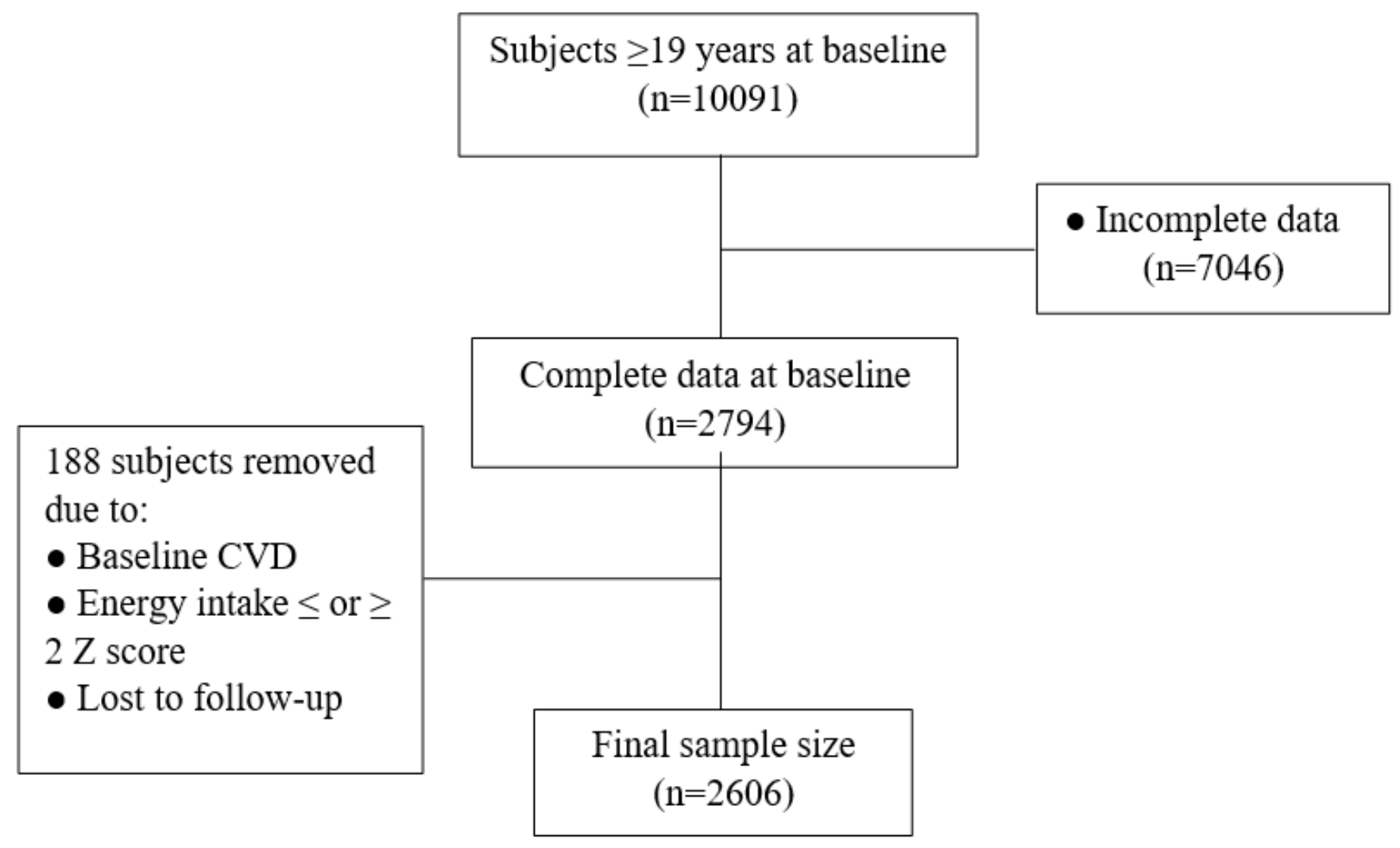

Figure 1 
Flow chart of study

Page 18/18 